\title{
Conceptual Design and Dynamics Testing and Modeling of a Mars Tumbleweed Rover
}

\author{
Philip C. Calhoun ${ }^{*}$ and Steven B. Harris ${ }^{\dagger}$ \\ NASA Langley Research Center, Hampton, Va., 23681 \\ Behzad Raiszadeh* \\ NASA Langley Research Center, Hampton, Va., 23681 \\ and \\ Kristina D. Zaleski ${ }^{\S}$ \\ Pennsylvania State University, University Park, Pa., 16802
}

\begin{abstract}
The NASA Langley Research Center has been developing a novel concept for a Mars planetary rover called the Mars Tumbleweed. This concept utilizes the wind to propel the rover along the Mars surface, bringing it the potential to cover vast distances not possible with current Mars rover technology. This vehicle, in its deployed configuration, must be large and lightweight to provide the ratio of drag force to rolling resistance necessary to initiate motion from rest on the Mars surface. One Tumbleweed design concept that satisfies these considerations is called the Eggbeater-Dandelion. This paper describes the basic design considerations and a proposed dynamics model of the concept for use in simulation studies. It includes a summary of rolling/bouncing dynamics tests that used videogrammetry to better understand, characterize, and validate the dynamics model assumptions, especially the effective rolling resistance in bouncing/rolling dynamic conditions. The dynamics test used cameras to capture the motion of 32 targets affixed to a test article's outer structure. Proper placement of the cameras and alignment of their respective fields of view provided adequate image resolution of multiple targets along the trajectory as the test article proceeded down the ramp. Image processing of the frames from multiple cameras was used to determine the target positions. Position data from a set of these test runs was compared with results of a three dimensional, flexible dynamics model. Model input parameters were adjusted to match the test data for runs conducted. This process presented herein provided the means to characterize the dynamics and validate the simulation of the EggbeaterDandelion concept. The simulation model was used to demonstrate full scale Tumbleweed motion from a stationary condition on a flat-sloped terrain using representative Mars environment parameters.
\end{abstract}

\begin{tabular}{ll} 
& \multicolumn{1}{c}{ Nomenclature } \\
$A^{R B}$ & $=$ Attitude transformation from body-fixed to room-fixed reference frame \\
$a_{i}$ & $=$ Weighting factors used for least squares solution \\
$B A R T$ & $=$ Basic Aerodynamic Research Tunnel \\
$C G$ & $=$ Center of Gravity \\
$D O F$ & $=$ Degrees of Freedom \\
$d_{L}$ & $=$ Axial deformation of the Eggbeater leg \\
$F_{L}$ & $=$ Axial Force applied to the Eggbeater leg during axial deformation
\end{tabular}

\footnotetext{
*Aerospace Engineer, Exploration Systems Engineering Branch, Mail Stop 365, AIAA Member

${ }^{\dagger}$ Aerospace Engineer, Exploration Systems Engineering Branch, Mail Stop 365

* Aerospace Engineer, Exploration Systems Engineering Branch, Mail Stop 365, AIAA Member

$\S$ Undergraduate Student, Aerospace Engineering Dept.
} 


$$
\begin{array}{ll}
J & =\text { Quadratic Cost Function } \\
\vec{P}_{C 0}^{R} & =\text { Position of the CG in the room-fixed frame expressed in room-fixed coordinates } \\
\vec{P}_{T_{i} 0}^{R} & =\text { Position of the ith Target location in the room-fixed frame expressed in room-fixed coordinates } \\
\vec{P}_{T_{i} C}^{B} & =\text { Position of the ith Target relative to the CG expressed in body-fixed coordinates } \\
\vec{P}_{T_{i} T_{j}}^{B} & =\text { Position of the ith Target relative to the jth Target expressed in body-fixed coordinates } \\
\vec{v} & =\text { Relative Target Position vectors expressed in room-fixed coordinates } \\
\vec{w} & =\text { Relative Target Position vectors expressed in body-fixed coordinates }
\end{array}
$$

\section{Introduction}

$\mathrm{T}_{\mathrm{s}}^{\mathrm{H}}$ HE NASA Langley Research Center has been developing a novel concept for a Mars planetary rover called the Mars Tumbleweed. ${ }^{1,2}$ This rover concept utilizes in-situ Martian winds to roll and bounce the rover across the Mars surface. Since the power to propel the vehicle is derived from the in-situ environment this vehicle concept has the potential to cover vast regions of the Mars terrain not possible with current Mars rover technology. The longrange mobility could be augmented with some degree of control, allowing the Tumbleweed to transport science instruments to specific sites over a vast region of the Martian surface. This capability may enhance or even enable surface missions for surveying and scouting large areas to, for example, search for life. A prior study identified the "Search for Life" goal as "the most scientifically interesting and challenging mission for the Tumbleweed concept". ${ }^{2}$ "In this role, Tumbleweed rovers would survey vast regions the planet's surface to locate areas of interest for follow-on, comprehensive surveys by future landers, rovers or perhaps human explorers". ${ }^{2}$ These considerations motivated the design of a novel Tumbleweed concept described in this paper.

This paper describes some basic mechanical design considerations used as a basis to develop a Tumbleweed design concept called the "Eggbeater-Dandelion". A 6DOF simulation used a dynamics model of this concept. A rolling/bouncing test of a scale model of the Eggbeater structural concept used videogrammetry to measure the motion providing data for validating model assumptions. The simulation model was used to demonstrate full scale Tumbleweed motion from a stationary condition on a flat-sloped terrain using representative Mars environment parameters.

\section{Eggbeater-Dandelion Tumbleweed Concept Design}

The original Dandelion Tumbleweed notional concept identified in the early Tumbleweed studies motivated the Eggbeater-Dandelion Tumbleweed concept. ${ }^{1}$ Four notional Tumbleweed concepts were described in this report, the Box Kite, the Dandelion, the Tumblecup, and the Sphere. These four concepts represent two broad design categories, 1) spoke-type structure, or 2) cage-type structure. The spoke-type structure is characterized by central core with "spoke" type legs that provide load bearing mechanical isolation for the core and mechanical support for drag producing surfaces. The Dandelion and Tumblecup are examples of these. The cage-type structure utilizes an outer shell structure or "cage" to carry loads and tension-load-bearing structure, such as cables, to support the core. The Box-Kite and Sphere are examples of these. The Spoke-type legs make it easier to analyze the load path to the core for both structural design sizing and dynamics modeling. In addition, they provide a convenient architecture for incorporating a centrally configured set of control effectors and sensors. Therefore spoke-type design was chosen to further mature as a Tumbleweed rover concept in this study.

The Tumbleweed rover, in its deployed configuration, must be large and lightweight to provide the proper ratio of drag force to rolling resistance necessary to initiate motion from rest on the Mars surface in moderate winds. The structural design must also be flexible enough to limit transient loading during occasional high-speed impact with Mars terrain. For small deformations the structure should be somewhat stiff, providing a small contact surface to reduce rolling resistance. For large deformations the structure needs to "soften" to absorb the impact energy and limit the peak g-load to the core. These considerations require a highly flexible design with somewhat non-linear softening stiffness characteristics for large deformations.

Design analysis showed that the radial legs of the original Dandelion and Tumblecup concepts have a structural buckling problem. The straight structure of the Dandelion legs was replaced with curved beam structure as shown in Fig. 1. Each leg resembles an eggbeater or whisk, hence is given the name "Eggbeater leg" or simply "Eggbeater". The complete leg assembly, which constitutes a new Tumbleweed concept, is referred to as the "Eggbeater- 
Dandelion". The structural configuration of the Eggbeaters has a softening-type radial stiffness characteristic as measured in load/deformation tests performed on a dynamics test article, described in Section III. Each leg consists of a number of curved beam elements called "Eggbeater ribbons". The width of each ribbon was tapered radially from the core attachment point to provide additional drag producing surface area. The three and five ribbon combination allows interlocking of the ribbons of adjacent Eggbeater legs to enable more drag surface area without leg-to-leg physical interference and may also enable easier radially compacted stowage. To reduce weight, each ribbon may be constructed with lightweight sail material with a structural load-carrying member along each ribbon edge. Each multi-functional leg provides a softening structure, drag producing sails, and, if embedded with actuation and sensing devices, a control effector. To maintain spherical symmetry, the configuration included thirtytwo legs with attachment points coincident with the vertices of the two largest Platonic solids, the Dodecahedron (20 vertices) and the Icosahedron (12 vertices). Figure 2 shows the Eggbeater-Dandelion design geometry with the core as a truncated Icosahedron, which resembles a soccer ball.

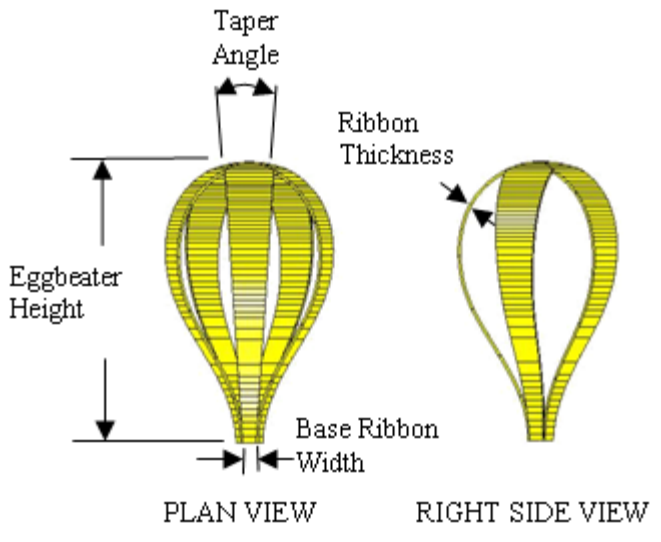

Figure 1. Eggbeater Leg Design

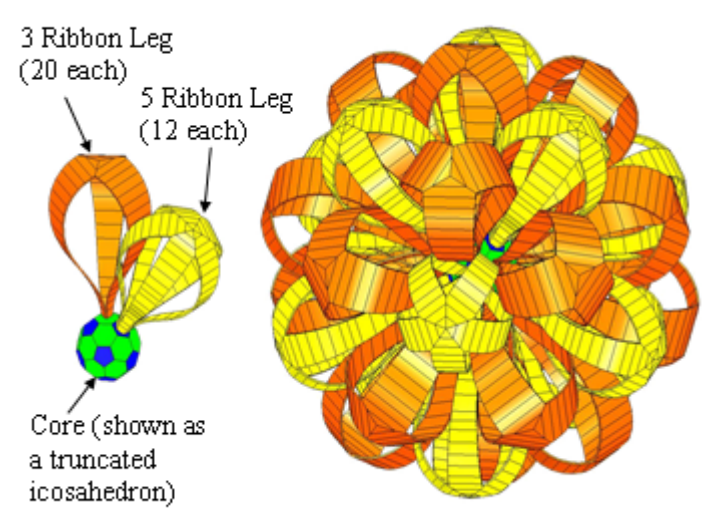

Figure 2. Eggbeater-Dandelion Design Configuration

A parametric model of this design was created using the I-deas Master Series CAD software. ${ }^{3}$ A fully constrained assembly drawing was created allowing rapid parametric changes to core diameter, eggbeater height, base ribbon width, ribbon taper angle and ribbon thickness. Table 1 includes a list of approximate dimensions of the Eggbeater-Dandelion concept parameters for the Mars rover application. These

\begin{tabular}{|l|l|}
\hline Parameter & Nominal Sizing \\
\hline Core diameter & $1.0 \mathrm{~m}$ \\
\hline Eggbeater height & $2.5 \mathrm{~m}$ \\
\hline Ribbon taper angle & $3-7 \mathrm{deg}$ \\
\hline
\end{tabular}

Table 1. Nominal Parameters for Mars Mission parameters correspond to a fully deployed Tumbleweed rover of an approximate diameter equal to $6 \mathrm{~m}$. This size was determined in initial feasibility studies to ensure mobility from rest in moderate Martian winds as well as to maneuver past obstacles such as rocks. ${ }^{2}$ The base ribbon width and ribbon thickness parameters are not specified at this point in the conceptual design. This parametric CAD model was used to mature the design/configuration and to create designs for wind tunnel models for aerodynamic testing conducted in the NASA Langley BART wind tunnel. ${ }^{4}$

\section{Dynamics Test using Videogrammetry}

A test of the dynamics of a Eggbeater-Dandelion Tumbleweed structure was conducted to characterize tumbling motion and to provide data to validate the assumptions made in a simulation model. Understanding the effects of structural non-linearity and damping on rolling resistance in bouncing/rolling dynamic conditions was of particular interest in this examination. The dynamics test used videogrammetry of targets affixed to a 34 in. diameter scaled test article representing the Eggbeater-Dandelion structural concept. The test article was bounced and rolled along a $24 \mathrm{ft}$ inclined ramp. Cameras monitored the dynamics of the test article by capturing the motion of 32 targets mounted to the article's outer structure. The estimated C.G. position was compared with results of a three dimensional, flexible dynamics simulation model. Model input parameters were adjusted to match the test data for various runs conducted. The test data along with the simulation work presented herein provided allowed us to characterize the dynamics and validate the simulation model assumptions for the Eggbeater-Dandelion concept. 


\section{A. Eggbeater-Dandelion Dynamics Test Article}

A simplified scale model of the Eggbeater-Dandelion concept was developed to better understand the rolling and bouncing dynamics as well as to validate a simulation model. Tests also sought to understand the effects of structural stiffness non-linearity and damping on rolling resistance in bouncing/rolling dynamic conditions.

The test article represented the Eggbeater-Dandelion concept structure. It was designed to approximate the structural flexibility of the full scale vehicle. The legs were built from loops cut from a coil of 5/8 in. width flat spring steel of 0.020 in. gauge. Each Eggbeater leg consisted of two loops of steel attached to a small wooden spool with a metal hose clamp at one end and tied together with a small piece of wire at the other end. The ridges on the ends of the spool provided a good surface to mount the ends of the spring steel loops. As the hose clamp was tightened the spring steel ends were bent and a preload was applied at the spool ends to ensure structure integrity of Eggbeater leg. The assembled Eggbeater leg, used for the prototype test article, is shown in Fig. 3.

Eggbeater legs of various sizes were constructed to determine their axial stiffness characteristics. The appropriate Eggbeater height for the legs used in the test article was chosen to be consistent with an estimate of the required test article system stiffness, $5 \mathrm{lb}$./in.. This system stiffness corresponds to an approximate rolling resistance coefficient in the range from 0.1-0.2. This determined the final design choice for the Eggbeater leg height, 15 inches. The measured load versus deflection for the $15 \mathrm{in}$. leg demonstrated a softening-type stiffness characteristic, as shown in Fig. 4. The leg was deflected in half-inch increments and the force was recorded at each increment for a range of deflection up to 8 inches.. From these data, curves of best fit were determined from linear up to a fourth order polynomial. The linear and fourth order curve fit equations are shown in Fig. 4, and given in Eq. (1) and (2), respectively.

$$
\begin{gathered}
F_{L}=0.8718 d_{L} \\
F_{L}=-0.0039 d_{L}{ }^{4}+0.0784 d_{L}{ }^{3}-0.5151 d_{L}{ }^{2}+1.9653 d_{L}
\end{gathered}
$$

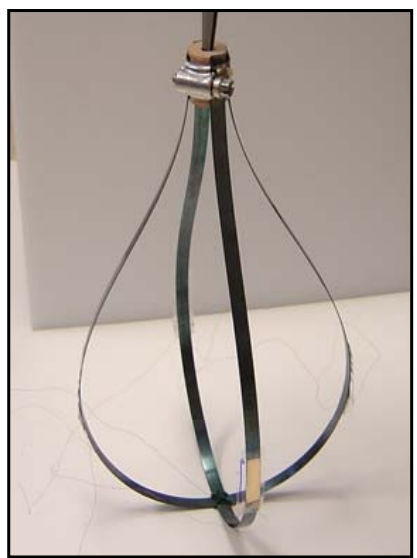

Figure 3. Eggbeater Prototype

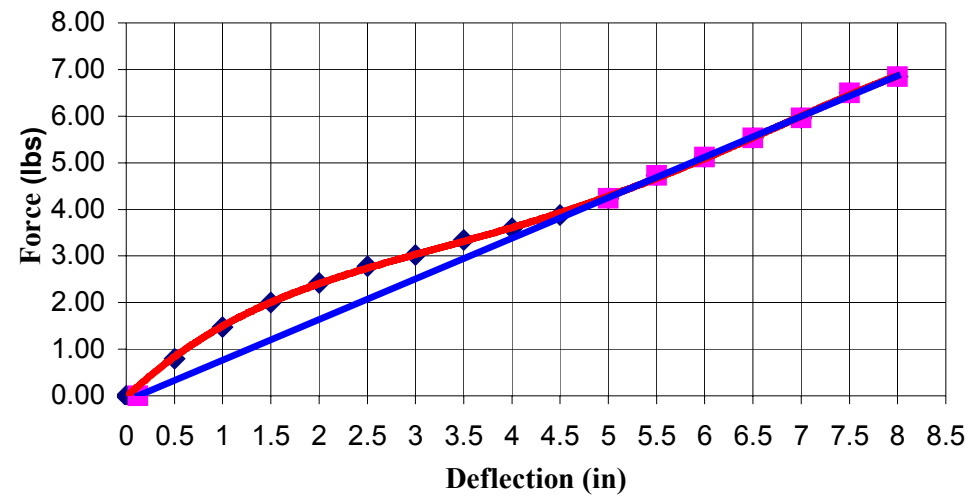

Figure 4. Measured Force versus deflection for a $15 \mathrm{in.} \mathrm{leg}$

The complete Eggbeater-Dandelion was built from 32 Eggbeater legs attached to the core using the compound Dodecahedron-Icosahedron pattern described for the full scale design in the previous section. Each Eggbeater leg was attached to the core, a regulation softball, using a $17 / 8 \mathrm{in}$. wood screw. The final constructed Eggbeater-Dandelion prototype test article, shown in Fig. 5, weighed $6 \mathrm{lb}$. Close examination of Fig. 5 reveals yellow and white plastic ties looped around the ends of the Eggbeater legs at the core attachment points. These ties were used to distinguish between the Dodecahedron and Icosahedron vertices and to serve as a safety device in the event of hose clamp failure.

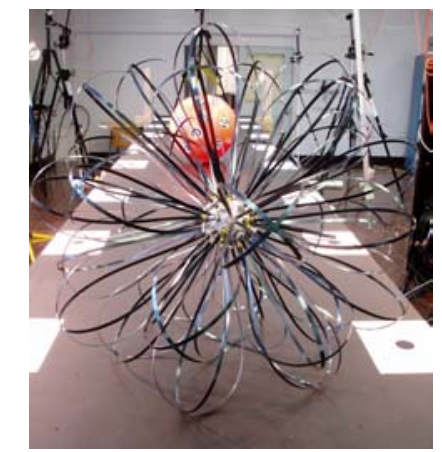

Figure 5. Eggbeater-Dandelion Test Article 


\section{B. Videogrammetry Test Configuration and Results}

The dynamics test consisted of releasing the Eggbeater-Dandelion test article onto a $24 \mathrm{ft}$ long ramp and capturing the motion of 32 coded targets affixed to the test article's outer structure with a set of video cameras. Five cameras were arranged so that targets on both sides of the test article would be visible from three distinct camera views at all times as the test article progressed down the ramp. The video output from each camera was hooked to a central computer that extracted the video frame data for frame-by-frame image processing. Videogrammetry was used to resolve each visible target's 3-dimensional location in a room-fixed reference frame. The process required at least three targets to be visible in any camera frame. To ensure a good videogrammetry solution at all times, one camera was placed on each side of the ramp near the top to capture good images at the beginning of the run and another pair of cameras further along the ramp to capture the later portion of the run. The fifth camera was stationed above the starting point of the ramp, to capture the top of the test article throughout the run. The targets were coded so that they would be easily distinguished from each other by the videogrammetry image processing software. Targets were also placed on the ramp to locate the test article on the ramp. Figure 6 shows the test setup with the coded targets applied to both the test article and the ramp. The test set-up also included various light fixtures used to illuminate the targets. The number, location, and orientation of these lights proved to be very important for capturing good video images with high contrast and low glare.

The ramp, constructed of three $4 \times 8$ ft. sections of plywood, was balanced in the middle of its length with a steel bar and held at the ends by four wooden uprights. This design permitted easy adjustment of the ramp angle so that the angle of inclination could be varied for different runs.

Three test articles were examined during the testing. The first test article, a simple sphere, was used to calibrate the test. The other two test articles were the Eggbeater-Dandelion and the Box-Kite ${ }^{1,2}$. A total of 107 runs were performed during testing; approximately 65 of those were of the EggbeaterDandelion test article. The test articles were rolled, shoved, and thrown down the ramp for ramp inclinations from 2-5 deg to

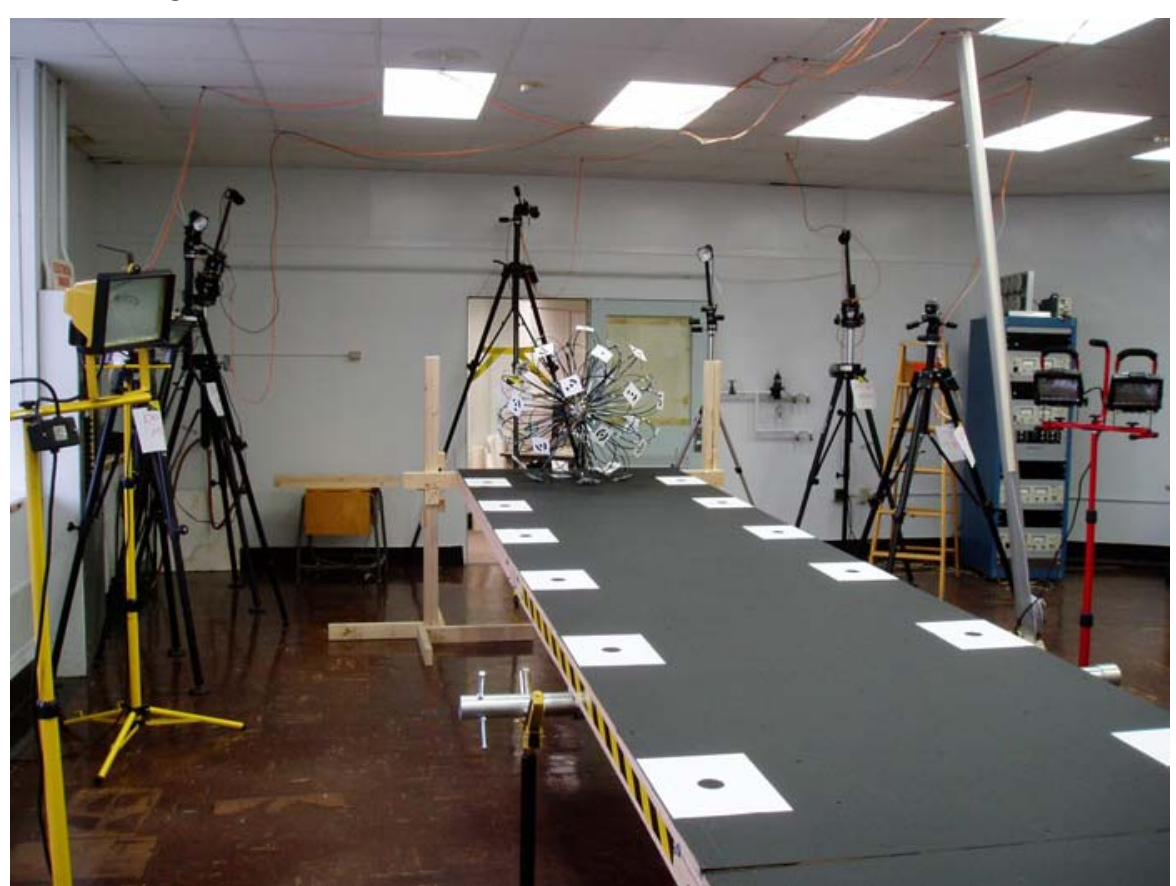

Figure 6. Eggbeater-Dandelion Prototype Model provide a range of test conditions. The analysis of the Box kite and sphere runs are not included in this paper since they are considered to be outside the scope of this work.

At least four good runs of the Eggbeater-Dandelion test article were completed at each test condition in order to obtain a good set of data. During set up runs, the lighting in the lab was adjusted to determine the optimal conditions for the test. The camera gain and exposure time were also varied since the flexible dynamics of the Eggbeater legs caused blurring of the target images.

\section{Test Data Reduction}

The test data collected in the video frame grabber was reformatted using $\mathrm{XnView}^{5}$. This reformatted data was input into the PhotoModeler ${ }^{6}$ software, which performs three-dimensional videogrammetry solutions. PhotoModeler has two different modes in which it can process target data forward in time. The first method requires the user to manually mark points to be tracked, and then the software searches the consecutive frames for those points. Using this method results in a loss of accuracy if the points are attached to a body in motion, so this method was only used to mark the stationary points along the ramp. The second method uses a set of coded targets that the software can automatically recognize and mark in the consecutive frames. PhotoModeler searches through all frames, then identifies and computes the image centriod for each coded target. This method was used for target identification on 
the Eggbeater-Dandelion test article since specific targets come into and out of view while the test article bounces and rolls along the ramp.

The software system includes twenty-five unique coded targets to place on the test article, which the software can distinguish. Figure 7 shows two examples of these coded targets. To capture the dynamics of the EggbeaterDandelion test article, targets were placed at the end of each Eggbeater leg. Since the Eggbeater test article has thirty-two legs and only twenty-five unique coded targets were used, the remaining legs were marked with targets, similar to the ones attached to the ramp.

Some difficulties were experienced using the PhotoModeler tracking system. The contrast had to be adjusted so that the maximum number of targets could be identified. But, by changing this setting, the PhotoModeler software would incorrectly identify other objects in the frame data as targets. Those points had to be removed from each frame by hand. Another quirk of PhotoModeler was incorrectly identifying coded targets. The system would sometimes try to identify a target at an extreme angle of incidence. At these large angles the tracking software occasionally incorrectly identified the coded targets. This was due to target image blurring from non-optimum lighting, or to glaring or shadowing of the image. The solution to this problem was in the nature of the target codes. Targets corresponding to low numbered codes, i.e. 1 and 2, were less complex than the targets corresponding to higher numbered codes. If a more complex target were on the edge of a test article, the program would try to read it as a lower numbered target. By setting the program to display all the video frames containing the lower codes, one could visually see if the points were correctly marked and fix any miss-referenced points. Occasionally, the PhotoModeler software would see the table target behind the open, Eggbeater structure, see that the test article was present, and conclude that the table target must be in front of the test article. This produced some rather strange results, so each frame had to be checked by hand after the
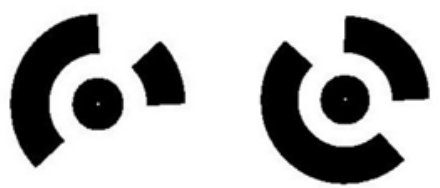

Figure 7. Coded Targets program had completed its analysis.

After each run had been processed in PhotoModeler, the target data was exported to a text file containing x, y, z coordinates of each identified target in a room-fixed reference frame for each frame of video. The software provided an error estimate for each target centroid, which was used to remove some target centriod solutions considered to be erroneous. For each frame of video data that contained at least three correctly identified targets, an estimate was made of the test article center of gravity for comparison with dynamics simulation results.

The first step in obtaining a CG estimate of the test article was to establish a body-fixed reference frame for the test article and resolve all 25 coded targets in each video frame. The body-fixed coordinates could then be used to provide an estimate of the body-fixed attitude of the test article relative to the room-fixed frame in order to obtain a CG estimate. The body-fixed coordinates for each coded target were determined by bringing together the information from each target as seen in the different camera views. This data was then processed in the PhotoModeler software, in a manner similar to that described previously, to obtain $\mathrm{x}, \mathrm{y}, \mathrm{z}$ coordinates of each coded target in an arbitrary room-fixed reference frame. Since the test article was held fixed in the room during the photography, this room-fixed reference frame was also fixed with respect to the test article. This then establishes the body-fixed reference frame locations of the targets.

The process for establishing a CG estimate for the Eggbeater-Dandelion assumed that the test article flexibility was negligible. Because the majority of the deformation during the test was from the legs that were in contact with the ground their attached targets were not visible to the PhotoModeler software and thus not used in the solution for the CG estimate. The remaining flexibility of the test article was accounted for by averaging solutions obtained from multiple sets of targets. Using the $\mathrm{i}^{\text {th }}$ identified target location the CG position of the test article can be expressed, as a sum of the target position and the transformed location of the body-fixed target location, as shown in Eq. (3).

$$
\vec{P}_{C 0}^{R}=\vec{P}_{T_{i} 0}^{R}+A^{R B} \quad \vec{P}_{T_{i} C}^{B}
$$

An estimate of the test article CG is obtained by averaging CG location solutions from all identified targets within each video frame. This process is repeated for all video frames that contain at least three targets. The position of the $\mathrm{i}^{\text {th }}$ identified target location, expressed in the room-fixed frame, was produced by videogrammetric data reduction. The position of the $i^{\text {th }}$ identified target location relative to the body CG, expressed in the body frame, is obtaining by averaging opposing pairs of target centriod locations obtained from the still photography as described above. This provides a good estimate of the CG location since the test article center, assumed to be coincident with the CG, is at exactly at the midpoint location of targets mounted to the ends of opposing pairs of Eggbeater legs. 
The test article attitude transformation from the body-fixed to the room-fixed frame needs to be determined in order to solve Eq. (3). Equations (4) and (5) define a set of relative target position vectors, expressed in the bodyfixed and room-fixed frames respectively, where $\mathrm{n}$ ranges from one to the number of unique pairs of identified target locations.

$$
\begin{aligned}
& \vec{v}_{n}=\vec{P}_{T_{i} C}^{B}-\vec{P}_{T_{j} C}^{B}, \text { for } i \neq j \\
& \vec{w}_{n}=\vec{P}_{T_{i} 0}^{R}-\vec{P}_{T_{j} 0}^{R}, \text { for } i \neq j
\end{aligned}
$$

Then, the matrix that transforms the vectors from Eq. (4) to those in Eq. (5) may be determined from one of several standard methods for attitude determination from vector measurements. Since these vectors were obtained from the videogrammetric process they are assumed to have some error. This error is a result the test article flexibility, target misalignment from the precise end of the Eggbeater legs, as well as the videogrammetry measurement error. An estimate of the attitude transformation that minimizes this error may be obtained by a weighted least squares solution that minimizes the quadratic cost function given in Eq. (6). The error estimate values obtained form the videogrammetry process are the basis for determining the weighting factors, $a_{i}$.

$$
J\left(A^{R B}\right)=\frac{1}{2} \sum_{i=1}^{n} a_{i}\left\|\vec{w}_{i}-A^{R B} \vec{v}_{i}\right\|^{2}
$$

References (7) and (8) describe the algorithm used to obtain the attitude transformation solution that minimizes Eq. (6). The method, called QUEST (for quaternion estimation), is cast in quaternion form and then solved as a maximum-likelihood solution. A good overview of this algorithm as applied to a similar application is given in Ref. (9).

\section{Dynamics Model and Test Comparison}

A lumped spring-mass type flexible dynamics simulation model of the Eggbeater-Dandelion was developed and coded in Matlab ${ }^{10}$. This model is used to demonstrate the wind-blown mobility of the full-scale Mars vehicle from a resting condition using a representative set of Eggbeater-Dandelion design and Mars environment parameters. Future dynamics studies of this Tumbleweed concept will include studies of wind-blown mobility along simulated Mars terrain at selected sites, and studies of controlled mobility using actuation of the Eggbeater legs including start/stop, downwind, and crosswind motion. This section describes the model and shows a comparison of simulated dynamics test results with processed results from a single run of the test article. A single run of the simulation model is conducted to demonstrate wind-blown mobility over flat Martian terrain.

\section{A. Dynamics Simulation Model}

The dynamics of the Eggbeater-Dandelion are modeled with a rigid core body and a flexible model for each Eggbeater leg. A spring type model with radial stiffness and damping was developed for each Eggbeater leg. Each leg is assumed to be flexible in translation only in the radial direction. All other degrees of freedom for each leg relative to the core attachment point, including rotational degrees of freedom, are assumed to be rigid. These assumptions simplified the initial model development and were justified by noting that the constructed Eggbeater leg was relativity stiff in rotational and transverse loading. The leg spring model included an option to implement nonlinear stiffness and damping or representative linear terms such as those determined during the Eggbeater leg compression tests (See Fig. 4). Ground contact forces for each leg are modeled as components normal and tangent to a locally flat, sloped surface. The normal force is computed as a component of the leg compression force. The tangential force is assumed to be simple coulomb friction with a stiction level near zero ground/leg relative motion. It is applied in a direction opposite the ground relative motion of each leg. Rotational friction of the leg/ground contact about the Eggbeater leg axis-of-symmetry is neglected in this model. The mass and inertia for each leg is lumped with the core rigid body and the flexibility of each leg is applied only when in contact with the ground. Legs not in contact with the ground are assumed to be rigid and extended radially at their respective equilibrium lengths. This simplified model seeks to capture the first order impact and rolling dynamics while neglecting high frequency modes due to individual leg vibrations. 


\section{B. Comparison of Simulation to Test}

The dynamics simulation model was exercised with the appropriate parameters and initial conditions to model a single dynamics test run of the Eggbeater-Dandelion test article. In this run the test article was tossed to a height of a few feet above the end of the ramp. For this test run the ramp was adjusted to a down-slope ramp angle of 2 deg. Estimates of the test trajectory points constructed from the processed video data are shown in Fig. 8. These estimates of the test article CG in room-fixed coordinates as viewed from the side of the ramp during the test were obtained from the reduced videogrammetry data in the manner described in the previous section. Also shown in Fig. 8 is the location of the ramp, shown in solid gray, and a datum shown at 17 inches above the ramp surface. Since this datum is equal to the undeformed test article radius it can be used to determine ground contact and leg compression during the test. The origin of the coordinate axes in Fig. 8 is approximately the location where the test article first contacts the ramp.

The mass properties of the test article were determined by measurement and estimates from the CAD model. The weight of the complete Eggbeater-Dandelion test article, shown in Fig. 5, was $6 \mathrm{lb}$. The rotational inertia of each Eggbeater leg about the core attachment point was determined from the I-deas ${ }^{3}$ parametric CAD model. These values were lumped together with the core body to provide an estimate the inertia of the complete Eggbeater-Dandelion test article. Since the core body was small in comparison to the overall test article, the core body and all the leg attachment hardware were assumed lumped together as a point mass with zero rotational inertia.

The initial velocity and location of the CG relative to the ramp was adjusted to match the initial CG trajectory points estimated from the test data. The initial rotational rate was nearly zero for this test run and thus neglected in the simulation

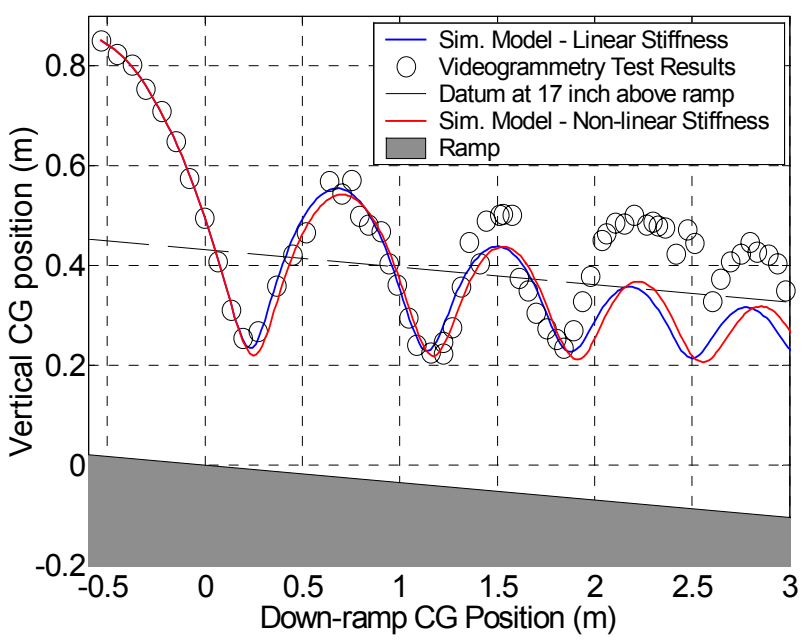

Figure 8. Simulation Comparison to a Dynamics Test Run model. Both a non-linear Eggbeater leg stiffness, based on the load/deformation measurements from the leg compression test shown in Fig. 4, and a linear approximation were used in separate simulation cases to model radial flexibility of the legs. The case with linear stiffness also included linear damping. The non-linear case utilized nonlinear damping proportional to the non-linear stiffness with the proportionality constant equal to the linear damping divided by the linear stiffness given in Eq. (1). These models provided nearly identical results and compared well with the Videogrammetry test results as shown in Fig. 8. In each case the damping term were tuned by trial-anderror until the height of each successive bounce best approximated the videogrammetry test results. The stiction and coulomb friction coefficients were tuned by trial-and-error until the down-ramp velocity after the first bounce nearly approximated the test results. Table 2 shows the parameters and initial conditions used in the simulation model. The reduced videogrammetric results show that the test article bounced several times as it tumbled down the ramp, as noted along the trajectory when the estimated test article CG height exceeds the 17-inch datum above the ramp. The simulated trajectory matches the videogrammetry test results very closely for the initial two bounces. Some scattering of the test data points about a smooth trajectory is noted, particularly after the first two bounces. Upon close examination of the video frame data this motion was attributed to high frequency vibration of the legs excited

\begin{tabular}{|c|c|c|c|c|}
\hline Initial Conditions & & & Model Parameters & \\
\hline CG Height above ramp & $0.85 \mathrm{~m}$ & & Mass & $6 \mathrm{lbm}$. \\
\hline CG Down-ramp location & $-0.53 \mathrm{~m}$ & & Inertia & $0.127 \mathrm{~kg} \mathrm{~m}{ }^{2}$ \\
\hline Vertical velocity & $-0.4 \mathrm{~m} / \mathrm{sec}$ & & Leg Stiffness - Linear Case & $214.2 \mathrm{~N} / \mathrm{m}$ \\
\hline Down-ramp velocity & $2.3 \mathrm{~m} / \mathrm{sec}$ & & Leg Damping - Linear Case & $1.57 \mathrm{~N} /(\mathrm{m} / \mathrm{s})$ \\
\hline Rotational rates & $0.0 \mathrm{deg} / \mathrm{sec}$ & & Leg Coulomb Friction Coef. & 0.36 \\
\hline & & & Leg Stiction Coef. & 0.47 \\
\hline
\end{tabular}

Table 2. Simulation Parameters 
by ground impact. After the third bounce the videogrammetry test results seem to indicate that the test article is bouncing above the ground. Examination of the video frame data shows that after the third bounce the test article remains in contact with the ground as is shown in the simulated trajectory results. Since the cameras used for the videogrammetry were located at the top end of the ramp it is assumed that the measured target location error grows as the test article moves toward the low end of the ramp. More processing test data is needed to understand the error in the videogrammerty. However, the simplified simulation model seems to capture the low frequency tumbling dynamics quite well.

\section{Evaluation of Wind-blown Mobility on Flat Martian Terrain}

The simulation model was used to evaluate the wind-blown mobility of the Eggbeater-Dandelion concept in a simulated Mars environment. This simulation study used the nominal system and environment parameters given in Table 3. These values are consistent with those determined in initial feasibility studies to ensure Tumbleweed mobility from rest in moderate Martian winds. ${ }^{2}$ The system diameter is consistent with the sizing parameters given in Table 1. The drag coefficient was set equal to an approximate value of a smooth sphere $(\mathrm{Cd}=0.5)$. This was considered to be a conservative lower bound based on the larger drag coefficients obtained for the Eggbeater concept in the BART wind tunnel tests. ${ }^{4}$ The values for the Mars gravity constant and atmospheric density are representative of surface conditions.

To demonstrate wind-blown mobility in representative wind conditions the Tumbleweed vehicle was initialized at rest in an equilibrium state on flat ground. A constant $8 \mathrm{~m} / \mathrm{sec}$ wind in the downrange direction was imparted to the vehicle. This is approximately the upper bound design wind for a flat slope used in the feasibility studies. ${ }^{2}$ Achieving the weight design goal of $10 \mathrm{~kg}$ for the full-scale vehicle is quite challenging. To minimize the impact on the structure weight the structural stiffness of each leg was given the same value as the scale model. Since most of the rolling resistance is due to structure damping the damping term for each Eggbeater leg was scaled by the double the amount of the mass increase of the full-scale vehicle. These leg parameters provided system flexibility equivalent to the scale model with a significant increase in damping. This increase provides a conservative estimate of the damping to account for increased mass and increased energy dissipation within the flexible tapered Eggbeater ribbons used on the full-scale Mars vehicle. The leg stiffness and damping values used in the simulation are shown in Table 3. Figure 9 shows the system CG trajectory for this case. The CG of the full-scale Eggbeater-Dandelion wobbles about its equilibrium state at a height of about $2.75 \mathrm{~m}$ above the surface while maintaining an approximate straight path downrange. Figure 10 shows the downrange velocity and rolling rate. This simulation demonstrates

\begin{tabular}{|c|c|c|c|}
\hline $\begin{array}{l}\text { Environment } \\
\text { Parameters }\end{array}$ & & $\frac{\text { System }}{\text { Parameters }}$ & \\
\hline Gravity constant & $3.71 \mathrm{~m} / \mathrm{s}^{2}$ & Diameter & $6 \mathrm{~m}$ \\
\hline Atmospheric density & $0.0155 \mathrm{~kg} / \mathrm{m}^{3}$ & Drag Coefficient & 0.5 \\
\hline Terrain Slope & $0 \mathrm{deg}$ & Leg Stiffness & $214.2 \mathrm{~N} / \mathrm{m}$ \\
\hline & & Leg Damping & $11.5 \mathrm{~N} /(\mathrm{m} / \mathrm{s})$ \\
\hline
\end{tabular}

Table 3. Simulation Parameters for Wind-blown Mobility Evaluation
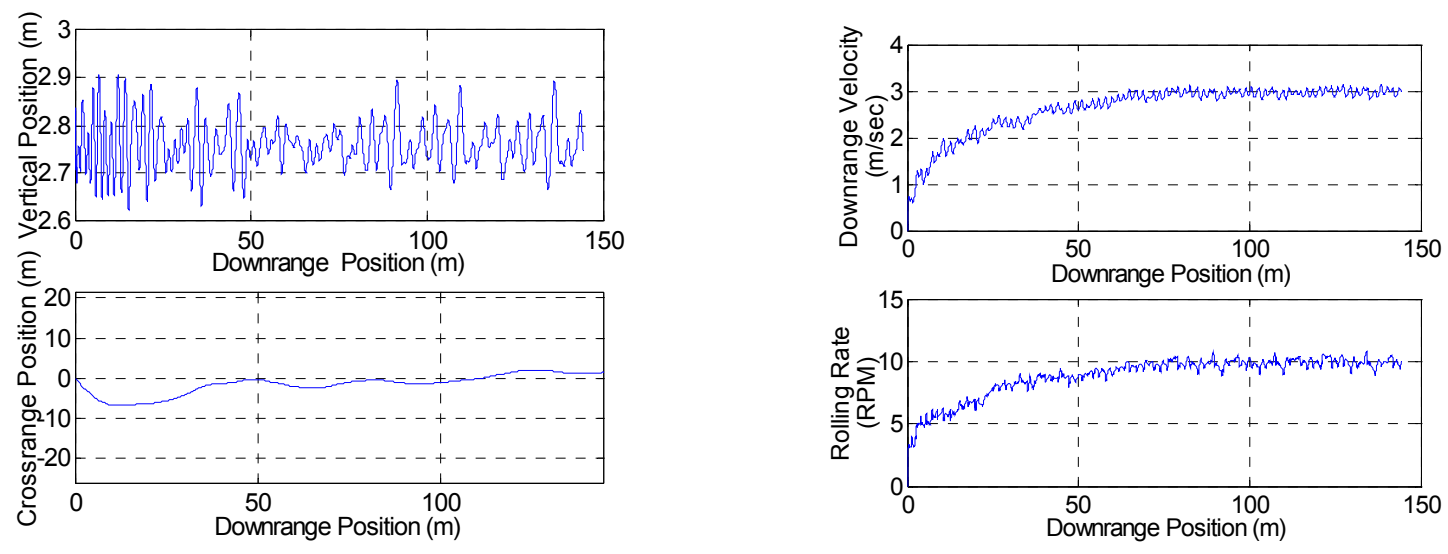

Figure 9. Wind-Blown Mobility - CG Position Figure 10. Wind-Blown Mobility - CG Velocity and Rate 
Eggbeater-Dandelion wind-blown mobility in moderate Mars surface wind conditions.

\section{Conclusions}

This paper has presented a new Tumbleweed concept called the Eggbeater-Dandelion, based on the original Dandelion notional concept from earlier studies. ${ }^{2}$ A prototype scaled test article was built for the purpose of characterizing the flexible dynamics of the rolling and bouncing motion. Dynamics tests using a videogrammertry were conducted and an estimate of the CG trajectory from a sample test run compared well with simulation model results. A lumped spring-mass type flexible dynamics simulation model of the Eggbeater-Dandelion was developed and coded in Matlab ${ }^{10}$. Rolling velocity results from the simulation using a set of representative Mars vehicle and environment parameters demonstrated wind-blown mobility on flat Martian terrain. This model will be used for various dynamics studies of this Tumbleweed concept, including studies of wind-blown mobility along simulated Mars terrain, and studies of controlled mobility using leg actuation. Future work should include development of prototype for field-testing on a Mars simulated terrain for the purpose of improving the characterization of the windblown mobility of this concept.

\section{Acknowledgments}

The authors would like to thank Tom Jones, Benny Lunsford, and Adrian Dorrington for providing, setting up, and operating the videogrammetry system, as well as providing assistance in the test data reduction. The authors would also like to thank to Jennifer Keyes, Greg Hajos, Jeff Antol, Chris Strickland, and Ji Su for constructing the test ramp and providing assistance in conducting the dynamics tests. Finally, the authors would like to acknowledge Jeff Antol and Greg Hajos for leading the project, and Dennis Bushnell for funding and supporting the project, under the NASA Langley Research Center's Creativity and Innovation program.

\section{References}

${ }^{1}$ Antol, J., Calhoun P. C., Flick, J., Hajos, G., Kolacinski, R., Minton, D., Owens, R., Parker, J., "Low Cost Mars Surface Exploration: The Mars Tumbleweed", NASA TM-2003-212411, Aug. 2003.

${ }^{2}$ Antol, J., Calhoun P. C., Hajos, G., Parker, J., and Stillwagen, F., "Mars Tumbleweed: FY2003 Conceptual Design Assessment", NASA TM-2005-XXXX, 2005.

${ }^{3}$ EDS I-deas 9, Computer Aided Design, Software Package, Ver. 9m2, EDS PLM Solutions, Milford, Oh., 2001.

${ }^{4}$ Strickland, C., and Parker, J., "Wind Tunnel Tests to Determine Drag Coefficients for the Mars Tumbleweed", AIAA-20050248, 43rd AIAA Aerospace Sciences Meeting and Exhibit, Reno, NV, January 10-13, 2005.

${ }^{5} \mathrm{XnView}$, Software Package, Ver. 1.70.2, Gougelet Pierre-emmanuel, France, 2004.

${ }^{6}$ PhotoModeler Pro 5, Measuring and Modeling the Real World, Software Package, Ver. 5.1.1, EOS Systems Inc, Vancouver, BC, Canada, 2004.

${ }^{7}$ Keat, J., “Analysis of Least-Squares Attitude Determination Routine, DOAOP”, Computer Sciences Corp., Report CSC/TM77/6034, Feb. 1977.

${ }^{8}$ Shuster, M. D. and Oh, S. D., "Three-Axis Attitude Determination from Vector Observations", Journal of Guidance and Control, Vol. 4, No. 1, pp. 70-77., Jan.-Feb. 1981.

${ }^{9}$ Calhoun, P. C. and Dabney, R.W., "A Solution to the Problem of Determining the Relative 6 DOF State for Spacecraft Automated Rendezvous and Docking", Proceedings of the SPIE (Society of Photo-Optical Instrumentation Engineers) - The International Society for Optical Engineering, Vol. 2466, pp 175-184, Orlando, Fl., April 17-18, 1995.

${ }^{10}$ Matlab, The Language of Technical Computing, Software Package, Ver. 6.5.0 Release 13, The MathWorks Inc, Natick, MA, 2002. 